\title{
Portrait des pratiques évaluatives des ergothérapeutes œuvrant au Québec
}

\author{
Overview of the assessment practices of occupational therapists \\ working in Quebec
}

\author{
Janie Gobeil $\odot$, Nadine Larivière, Annie Carrier, Nathalie Bier, Carolina Bottari, \\ Nathalie Veillette, Suzanne Rouleau, Isabelle Gélinas, Véronique Provencher, \\ Mélanie Couture et Mélanie Levasseur
}

Mots clés : Ergothérapie; Évaluation; Outils de mesure; Pratique professionnelle; Instruments de mesure.

Key words: Assessment; Assessment tools; Occupational therapy; Professional practice.

\begin{abstract}
Abrégé
Description. L'évaluation du client est primordiale dans la pratique de l'ergothérapie, notamment pour établir les priorités de traitement et pour vérifier l'efficacité des interventions. Les pratiques évaluatives des ergothérapeutes sont cependant peu documentées. But. Dresser un portrait des pratiques évaluatives des ergothérapeutes du Québec selon les dimensions personne-environnement-occupation et les clientèles. Méthodologie. Une enquête transversale a été réalisée à l'aide d'un sondage en ligne envoyé aux ergothérapeutes du Québec. Résultats. En pédiatrie, les ergothérapeutes utilisent majoritairement des outils standardisés des aptitudes physiques et neurologiques. L'évaluation d'adultes cible principalement les aptitudes physiques et la productivité. Auprès des aînés, l'évaluation repose surtout sur les aspects fonctionnels, particulièrement les aptitudes physiques, les soins personnels et la sécurité à domicile, et sur le dépistage des difficultés cognitives. Implications pour la pratique. L'évaluation ergothérapique serait généralement axée sur les aptitudes physiques. Pour assurer une approche holistique, l'occupation et l'environnement devraient être davantage considérés dans l'évaluation.
\end{abstract}

\begin{abstract}
Background. In occupational therapy practice, client assessments are essential for establishing treatment priorities and determining the effectiveness of interventions. However, occupational therapists' assessment practices are not well documented. Purpose. This work aimed to provide an overview of the assessment practices of Quebec occupational therapists based on the person-environment-occupation components and clienteles. Method. A cross-sectional survey was conducted using an online survey that was sent to occupational therapists in Quebec. Findings. In paediatrics, occupational therapists tend to use standardized tools to assess physical and neurological abilities. Adult assessment focuses mainly on physical abilities and productivity. For seniors, assessment focuses mainly on functional aspects (physical abilities, personal care, and home safety) and screening for cognitive difficulties. Implications. Occupational therapy assessment mostly focuses on physicial abilities. To ensure a holistic approach, more occupational and environmental components should be included in the assessment practices.
\end{abstract}

Financement : Janie Gobeil est actuellement boursière de la Faculté de médecine et des sciences de la santé de l'Université de Sherbrooke (20I8-20). Au moment de l'étude, Mélanie Levasseur était chercheuse boursière Junior I du Fonds de recherche du Québec-Santé (\#268I5; 2013-17). Elle est maintenant nouvelle chercheuse des Instituts de recherche en santé du Canada (\#360880; 2017-22).

Auteure ressource : Centre de recherche sur le vieillissement, Centre intégré universitaire de santé et de services sociaux (CIUSSS) de l'Estrie-Centre hospitalier universitaire de Sherbrooke (CHUS), 1036 rue Belvédère Sud, Sherbrooke, Québec, JIH 4C4, Canada. Téléphone : 8I9-780-2220 poste 45655. Courriel : Janie.Gobeil@USherbrooke.ca 
$\mathrm{P}$ our offrir des services d'ergothérapie efficients et de qualité, le processus d'évaluation du client est primordial. Ce processus sert notamment à établir les priorités de traitement, à poser un jugement sur le fonctionnement de personnes dans leurs activités courantes et leurs activités sociales et à documenter l'efficacité des interventions (Ordre des ergothérapeutes du Québec [OEQ], 2010). Afin de faire une analyse juste des informations recueillies, les ergothérapeutes doivent s'assurer d'utiliser des méthodes d'évaluation rigoureuses incluant des outils aux qualités psychométriques adéquates et axés sur la participation et l'engagement des personnes dans leurs occupations (OEQ, 2014).

Les pratiques évaluatives doivent par ailleurs être guidées par des modèles ergothérapiques, et ce, peu importe la clientèle (OEQ, 2016b; Association canadienne des ergothérapeutes [ACE], 2012). Par exemple, le Modèle canadien du rendement et de l'engagement occupationnels (MCREO; Townsend et Polatajko, 2013) illustre trois principales composantes devant être évaluées par les ergothérapeutes: (a) les dimensions physiques, affectives, cognitives et spirituelles de la personne (P); (b) les éléments physiques, sociaux, culturels et institutionnels de l'environnement $(\mathrm{E})$; et (c) les domaines de l'occupation $(\mathrm{O})$, soient les soins personnels, la productivité et les loisirs. Or, les méthodes et les outils d'évaluation pouvant être utilisés dans le cadre de ces modèles sont nombreux et en constante évolution. Les méthodes d'évaluation font référence aux modalités d'évaluation (ex.: observation, mises en situation, entrevues, etc.), alors que les outils sont davantage formels, incluant, par exemple, des procédures d'administration ou d'interprétation, et pour lesquels parfois les qualités métrologiques ont été étudiées ou des valeurs de référence ont été développées. Ainsi, afin de favoriser une pratique fondée sur les résultats probants (OEQ, 2014), il importe de mieux connaître sur quelles composantes du PEO portent les évaluations actuellement réalisées sur le terrain ainsi que les méthodes et les outils utilisés.

Quelques études menées au Canada et au Québec ont permis d'explorer les pratiques des ergothérapeutes. Réalisées dans différents contextes de pratique très spécifiques tels qu'à l'urgence, en santé mentale ou auprès de personnes ayant des déficits cognitifs, les études rapportaient généralement une grande variabilité des pratiques ergothérapiques (Belchior, Korner-Bitensky, Holmes et Robert, 2015; Douglas, Liu, Warren et Hopper, 2007; Korner-Bitensky, Barrett-Bernstein, Bibas et Poulin, 2011; Rouleau, Dion et Korner-Bitensky, 2015) ainsi qu'une large utilisation de méthodes d'évaluation non standardisées (Belchior et al., 2015; Douglas et al., 2007; Korner-Bitensky et al., 2011; Rouleau et al., 2015; Veillette, Demers et Dutil, 2007). Selon Douglas et collaborateurs (2007), les ergothérapeutes travaillant auprès des aînés présentant des atteintes cognitives utilisent une approche globale du fonctionnement, les tâches de la vie quotidienne et de cuisine étant leurs principales cibles d'évaluation. Dans l'étude de
Rouleau et collaborateurs (2015) sur la pratique en santé mentale adulte, les dimensions physique, socioculturelle et spirituelle étaient très peu évaluées. Quelques méthodes informelles étaient rapportées pour évaluer la productivité et l'environnement, mais presque aucun outil standardisé n'était utilisé. Auprès de la clientèle pédiatrique, les ergothérapeutes concentreraient davantage leurs pratiques sur les déficits et le fonctionnement dans les activités de la vie quotidienne (AVQ) et l'alimentation et moins sur le jeu, les loisirs et l'intégration sociale (Saleh et al., 2008). Enfin, selon les études canadiennes incluant la province du Québec, le choix des outils d'évaluation utilisés par les ergothérapeutes est notamment influencé par leur disponibilité dans leur milieu de travail, leur validité, leur fiabilité, leur temps et leur facilité d'administration (Belchior et al., 2015) et d'interprétation (Douglas et al., 2007). En bref, quelques études ont porté sur les pratiques d'ergothérapeutes œuvrant auprès de clientèles spécifiques, mais, à notre connaissance, aucune étude n'a réalisé un bilan des pratiques évaluatives de l'ensemble des ergothérapeutes du Québec, documentant uniformément les composantes évaluées et les méthodes et outils d'évaluation utilisés. Étant méconnues et susceptibles d'évoluer, notamment en raison des transformations survenues au cours des dernières années dans le réseau public de santé et le Code des professions du Québec, il est nécessaire de réaliser un bilan des pratiques évaluatives et de le comparer aux résultats probants. Puisque les ergothérapeutes du Québec représentent plus du quart $(28,0 \%)$ des ergothérapeutes canadiens (Institut canadien d'information sur la santé, 2015), un portrait de leurs pratiques pourrait guider les évaluations réalisées dans d'autres provinces, dans la francophonie et même ailleurs dans le monde. Ce portrait constituera également un point de référence pour l'observation de l'évolution des pratiques ergothérapiques. Enfin, les résultats guideront les chercheurs quant au développement d'outils d'évaluation dans les composantes où des lacunes sont identifiées. La présente étude visait donc à dresser un portrait global des pratiques évaluatives des ergothérapeutes du Québec en décrivant et en comparant, pour chaque clientèle selon leur groupe d'âge (petite enfance, enfants, adultes et aînés), la fréquence d'évaluation des composantes personne-environnementoccupation, définies selon le modèle MCREO, ainsi que les méthodes et les outils les plus utilisés pour les évaluer.

\section{Méthodologie}

\section{Dispositif, participants et procédure}

Pour répondre à cet objectif, une enquête transversale sur les pratiques d'évaluation et d'intervention des ergothérapeutes du Québec a été réalisée à l'été 2014 par l'OEQ auprès de tous ses membres admissibles. Au Québec, une personne qui souhaite pratiquer les activités professionnelles réservées par le Code des professions au champ d'exercice de l'ergothérapie doit détenir un permis valide et être inscrite au tableau de l'OEQ 
(Office des professions du Québec, 2018). Au total, 3725 ergothérapeutes répondaient aux critères d'admissibilité, soit d'avoir: (a) directement exercé des fonctions cliniques dans la dernière année et (b) transmis une adresse courriel à l'OEQ lors de leur inscription au tableau des membres. Ainsi, les membres de l'OEQ admissibles à participer à l'étude ont reçu le 30 juillet 2014 un courriel les invitant à répondre de façon volontaire et dans un délai de six semaines à un sondage en ligne sur le site de SurveyMonkey (San Mateo, CA, USA; www.surveymonkey.com). Au besoin, les ergothérapeutes pouvaient sauvegarder leurs réponses et poursuivre le remplissage plus tard. Deux rappels par courriel ont été envoyés approximativement aux deux semaines après le premier envoi.

\section{Sondage}

Le sondage a été conçu par une équipe d'ergothérapeutes comprenant 10 chercheurs et un directeur du développement et de la qualité de l'exercice de l'OEQ. Ce sondage était composé de trois sections; la première portant sur les pratiques d'évaluation, la seconde sur les pratiques d'intervention (non couvert dans le présent article) et la troisième sur les données sociodémographiques des répondants. La partie sur l'évaluation comportait deux principales questions. Tout d'abord, les ergothérapeutes devaient indiquer à quelle fréquence (voir ci-dessous) leurs évaluations étaient en lien avec la personne (P), l'environnement $(\mathrm{E})$ et l'occupation $(\mathrm{O})$. Afin de préciser davantage, chacune des composantes était détaillée en souscomposantes (ex. aptitudes physiques, environnement social, soins personnels, productivité, etc.). Pour assurer l'utilisation de termes connus des cliniciens et suivant les lignes directrices de l'OEQ, les terminologies de deux cadres théoriques largement utilisés en clinique au Québec, soit le MCREO (Townsend et Polatajko, 2013) et le Modèle du développement humain et du processus de production du handicap (MDH-PPH; Fougeyrollas, 2010), ont guidé la conception du sondage. Bien qu'ils utilisent une terminologie différente, ces deux modèles illustrent l'interaction de la personne à travers ses occupations et dans son environnement. Plus spécifiquement, le $\mathrm{P}$ du MCREO fait référence aux facteurs personnels du MDH-PPH, le E aux facteurs environnementaux et le $\mathrm{O}$ aux habitudes de vie. En s'inspirant des travaux de Richard, Gauvin, Gosselin et Laforest (2008), les fréquences auxquelles les ergothérapeutes rapportaient évaluer ces dimensions ont été converties en nombre de jours par mois (presque à tous les jours: 20; au moins une fois par semaine: 6 ; au moins une fois par mois: 2 ; moins d'une fois par mois: 1 ; jamais: 0 ) et la moyenne a été calculée pour chaque sous-composante. Ensuite, les ergothérapeutes devaient nommer les méthodes et les outils d'évaluation utilisés dans leur pratique selon leur fréquence d'utilisation (10 à 15 réponses maximum par fréquence). Les ergothérapeutes étaient invités à inscrire tout type d'évaluation en répondant, tel que spécifié, le plus près possible de la réalité, c'est-à-dire en indiquant ce qu'ils utilisaient et non ce qu'ils souhaiteraient utiliser. Les fréquences d'utilisation de ces méthodes et de ces outils ont également été converties en nombre de jours par mois. Enfin, des données sociodémographiques sur les ergothérapeutes (ex. âge, niveau de scolarité, situation d'emploi actuelle, etc.) ainsi que sur leur contexte de pratique, incluant le groupe d'âge de leur principale clientèle (c.-à-d. petite enfance: 0-4 ans; enfants: 5-17 ans; adultes: 18-64 ans; ou aînés: $65+$ ans), étaient recueillies.

\section{Analyses}

Les participants et les pratiques d'évaluation les plus souvent rapportées ont été décrits à l'aide de moyennes et d'écart-types pour les variables continues et de fréquences et de pourcentages pour les variables catégorielles. Des statistiques comparatives (test de Chi-2 et analyse de variance) ont été réalisées pour identifier les différences selon les clientèles, et ce, tant pour les caractéristiques des ergothérapeutes pouvant influencer les méthodes et les outils utilisés (ex.: nombre d'heures de formation par année, accès aux écrits scientifiques, etc.) que pour les composantes évaluées. Une valeur- $p$ plus petite que 0,05 était considérée significative. Les évaluations rapportées à la deuxième question ont été classées de manière à identifier: (a) les outils " formels ", c'est-à-dire un outil pouvant avoir un protocole d'administration, des normes, des qualités métrologiques, du matériel spécifique, etc. (ex. Peabody Developmental Motor Scales, Montreal Cognitive Assessment, Box and Block Test, etc.) et (b) tout autre méthode d'évaluation informelle ou " maison " (ex. mises en situation, observations, questionnaires, entrevues, etc.). La première auteure, alors étudiante en ergothérapie, a réalisé le classement qui a ensuite été révisé par huit chercheures ayant différentes expertises (ex. santé mentale, cognition, pédiatrie, etc.). Les logiciels SPSS et Microsoft Word ont été utilisés pour réaliser les analyses quantitatives et le classement des méthodes et des outils d'évaluation.

\section{Résultats}

\section{Caractéristiques des participants}

$\mathrm{Au}$ total, $1081(29,0 \%)$ ergothérapeutes ont répondu au sondage en ligne, dont $793(73,4 \%)$ ayant complété la question sur le groupe d'âge de leur clientèle principale. Ces participants sont similaires à l'ensemble des ergothérapeutes admissibles en termes de genre, d'âge et de groupe d'âge de clientèle. Les participants étaient majoritairement des femmes, âgés dans la trentaine, détenant un baccalauréat et travaillant à temps complet en milieu urbain (Tableaux 1 et 2). Les ergothérapeutes avaient en moyenne approximativement 13 à 14 années d'expérience clinique et intervenaient auprès de 11 à 15 clients par semaine. La majorité des répondants $(n=636$; $80,2 \%$ ) travaillait principalement auprès de la clientèle adulte 
Tableau I

Caractéristiques des ergothérapeutes par clientèle, variables continues

\begin{tabular}{|c|c|c|c|c|c|c|}
\hline & $\begin{array}{l}\text { Petite enfance } \\
\quad\left(n=62^{*}\right)\end{array}$ & $\begin{array}{c}\text { Enfants } \\
\left(n=95^{*}\right)\end{array}$ & $\begin{array}{c}\text { Adultes } \\
\left(n=307^{*}\right)\end{array}$ & $\begin{array}{c}\text { Aînés } \\
(n=329 *)\end{array}$ & Valour & $\begin{array}{c}\text { Tous } \\
(n=793 *)\end{array}$ \\
\hline Variable continue & $M(E ́ .-T)$. & $M(E ́-T)$. & $M(E ́-T)$. & $M(E ́ .-T)$. & & $M(E ́-T)$. \\
\hline $\begin{array}{l}\text { Expérience clinique (années) } \\
\text { Données } \\
\text { manquantes }\end{array}$ & $13,9(10,0)$ & $13,5(9,5)$ & $\begin{array}{c}12,8(9,7) \\
\mid\end{array}$ & $13,2(9,2)$ & 0,80 & $\begin{array}{c}13,1 \\
1\end{array}$ \\
\hline $\begin{array}{l}\text { Formation continue } \\
\text { (\# d'heures/année)** } \\
\text { Données } \\
\text { manquantes }\end{array}$ & $\begin{array}{l}64,3(150,1)^{a} \\
I\end{array}$ & $\begin{array}{c}36,8(34,1) \\
2\end{array}$ & $\begin{array}{c}37,4(124,8) \\
6\end{array}$ & $\begin{array}{l}24,2(35,5) \\
5\end{array}$ & 0,01 & $\begin{array}{c}33,9(92,3) \\
14\end{array}$ \\
\hline $\begin{array}{l}\text { Stagiaires (\#/année)** } \\
\text { Données } \\
\text { manquantes }\end{array}$ & $2,9(2,9)$ & $2,5(3,2)$ & $\begin{array}{l}2,9(4,4) \\
5\end{array}$ & $\begin{array}{l}2,2(3,0) \\
7\end{array}$ & 0,09 & $\begin{array}{l}2,5(3,6) \\
12\end{array}$ \\
\hline
\end{tabular}

Note. 288 participants n'ont pas indiqué à quel groupe d'âge leur principale clientèle appartenait.

*Sauf lorsqu'indiqué. **Au cours des 5 dernières années.

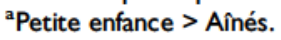

ou aînée (Tableaux 1 et 2). Comparativement à ceux travaillant auprès d'autres clientèles (plus particulièrement la clientèle aînée), les ergothérapeutes œuvrant en petite enfance rapportaient avoir réalisé un nombre supérieur d'heures de formation continue par année au cours des cinq dernières années. Les ergothérapeutes travaillant auprès des aînés auraient moins accès aux écrits scientifiques que ceux auprès d'autres clientèles (Tableaux 1 et 2).

\section{Composantes personne-environnement- occupation (P-E-O) évaluées par les ergothérapeutes}

Globalement, les aptitudes physiques étaient la souscomposante la plus évaluée par les ergothérapeutes, soit environ aux deux jours, tandis que la spiritualité était la moins évaluée, soit approximativement une fois par mois (Tableau 3). Les sous-composantes étaient évaluées selon des fréquences différentes en fonction des clientèles, à l'exception des aptitudes sociales et culturelles qui étaient en moyenne évaluées approximativement une fois par semaine. Comparativement aux autres clientèles, les jeux et les loisirs étaient plus fréquemment évalués chez les jeunes enfants, la productivité étaient plus fréquemment évaluée chez les adultes et les soins personnels étaient plus fréquemment évalués chez les aînés (Tableau 3). Les enjeux et les défis occupationnels en tant que souscomposantes de l'occupation étaient plus fréquemment évalués auprès de la clientèle adulte que des autres clientèles. Enfin, comparativement aux autres clientèles, l'environnement, autant physique que social, était plus fréquemment évalué chez les aînés (Tableau 3).
Plus spécifiquement pour la clientèle petite enfance, et considérant l'ensemble des résultats, les aptitudes physiques, les jeux et les loisirs et les aptitudes cognitives et neurologiques étaient plus souvent évalués (Tableau 3). Chez les enfants, il s'agissait des aptitudes physiques, des aptitudes cognitives et neurologiques et des jeux et des loisirs. Pour la clientèle adulte, les aptitudes physiques, la participation, de façon générale, et la productivité étaient plus souvent évaluées (Tableau 3). Enfin, chez les aînés, les soins personnels, les aptitudes physiques et l'environnement physique étaient les plus souvent évalués. Ainsi, alors qu'elle portait principalement sur des souscomposantes de la personne chez les enfants $(P=2 / 3)$ et de l'occupation chez les adultes $(O=2 / 3)$, l'évaluation des ergothérapeutes touchait les trois composantes chez les aînés (P-E-O; Tableau 3).

\section{Méthodes et outils d'évaluation utilisés par les ergothérapeutes}

Au total, 562 méthodes et outils d'évaluation différents ont été rapportés par les ergothérapeutes (données non présentées en tableau). Parmi tous les répondants, près du tiers $(n=279$; $32,0 \%$ ) ont rapporté utiliser les mises en situation ou l'observation (Petite enfance: $n=12 ; 19,7 \%$, Enfants: $n=$ $23 ; 25,6 \%$, Adultes: $n=92 ; 31,1 \%$, Aînés: $n=126,39,3 \%$ ). De plus, plus d'un cinquième $(n=196 ; 22,4 \%)$ des participants évaluaient à l'aide de méthodes dites " maisons " (Petite enfance: $n=11 ; 18,0 \%$, Enfants: $n=10 ; 11,1 \%$, Adultes: $n=71 ; 24,0 \%$, Aînés: $n=85 ; 26,5 \%$ ). Alors qu'une très faible proportion $(n=41 ; 4,7 \%)$ des ergothérapeutes ont explicité utiliser le MCREO lors de leur évaluation, cette proportion est similaire à ceux qui $(n=61 ; 7,0 \%)$ ont 
Tableau 2

Caractéristiques des ergothérapeutes par clientèle, variables catégorielles

\begin{tabular}{|c|c|c|c|c|c|c|}
\hline \multirow[b]{2}{*}{ Variable catégorielle } & \multirow{2}{*}{$\begin{array}{l}\begin{array}{l}\text { Petite enfance } \\
\left(n=62^{*}\right)\end{array} \\
n(\%)\end{array}$} & \multirow{2}{*}{$\begin{array}{c}\begin{array}{c}\text { Enfants } \\
\left(n=95^{*}\right)\end{array} \\
n(\%)\end{array}$} & \multirow{2}{*}{$\frac{\begin{array}{c}\text { Adultes } \\
\left(n=307^{*}\right)\end{array}}{n(\%)}$} & \multirow{2}{*}{$\frac{\begin{array}{c}\text { Aînés } \\
(n=329 *)\end{array}}{n(\%)}$} & \multirow[t]{2}{*}{ Valeur $p$} & \multirow{2}{*}{$\begin{array}{c}\begin{array}{c}\text { Tous } \\
\left(n=793^{*}\right)\end{array} \\
n(\%)\end{array}$} \\
\hline & & & & & & \\
\hline Genre & & & & & 0,28 & \\
\hline Féminin & $60(98,4)$ & $90(95,7)$ & $283(92,2)$ & $306(93,3)$ & & $739(93,5)$ \\
\hline $\begin{array}{l}\text { Données } \\
\text { manquantes }\end{array}$ & I & 1 & & 1 & & 3 \\
\hline $20-29$ & $16(25,8)$ & $24(25,3)$ & $97(31,6)$ & $87(26,4)$ & & $224(28,2)$ \\
\hline $30-39$ & $23(37,1)$ & $39(4 I, 1)$ & $98(31,9)$ & II I $(33,7)$ & & $271(34,2)$ \\
\hline $40-49$ & $13(21,0)$ & $18(18,9)$ & $68(22,1)$ & $84(25,5)$ & & $183(23,1)$ \\
\hline $50-59$ & $9(14,5)$ & $13(13,7)$ & $42(13,7)$ & $43(13,1)$ & & $107(13,5)$ \\
\hline $60-69$ & I $(1,6)$ & $\mathrm{I}(\mathrm{I}, \mathrm{I})$ & $2(0,7)$ & $4(1,2)$ & & $8(1,0)$ \\
\hline Niveau de scolarité & & & & & 0,16 & \\
\hline Situation d'emploi & & & & & 0,02 & \\
\hline Temps plein & $39(63,9)$ & $61(64,9)$ & $236(77, I)^{\mathrm{b}, \mathrm{c}}$ & $221(67,8)$ & & $557(70,8)$ \\
\hline $\begin{array}{l}\text { Données } \\
\text { manquantes }\end{array}$ & I & I & I & 3 & & 6 \\
\hline \multicolumn{7}{|l|}{ Établissement** } \\
\hline $\begin{array}{l}\text { CRDP } \\
\text { CRDITED }\end{array}$ & $\begin{array}{l}31(50,0) \\
\text { II }(17,7)\end{array}$ & $38(40,0)$ & $89(29,3)$ & & & $\begin{array}{c}184(23,3) \\
27(3,4)\end{array}$ \\
\hline $\mathrm{CP}$ & & $16(16,8)$ & $76(25,0)$ & & & $95(12,0)$ \\
\hline CLSC & & & & $113(34,5)$ & & $173(21,9)$ \\
\hline $\mathrm{CH}$ & & & & $76(23,2)$ & & III $(14,1)$ \\
\hline Troubles liés au vieillissement & & & $65(21,2)$ & $294(89,4)$ & & $361(45,5)$ \\
\hline Démences et syndromes associés & & & & $284(86,3)$ & & $338(42,6)$ \\
\hline Milieux & & & & & 0,05 & \\
\hline Urbain ( $\geq 150000$ habitants) & $33(53,2)$ & $50(53,2)$ & $187(6 \mid, 5)$ & $179(54,9)$ & & $449(57,1)$ \\
\hline Rural (<150 000 habitants) & $15(24,2)$ & $19(20,2)$ & $71(23,4)$ & $96(29,4)$ & & $201(25,6)$ \\
\hline Mixte**** & $14(22,6)$ & $25(26,6)$ & $46(15,1)$ & $51(15,6)$ & & $136(17,3)$ \\
\hline $\begin{array}{l}\text { Données } \\
\text { manquantes }\end{array}$ & & 1 & 3 & 3 & & 7 \\
\hline \multicolumn{7}{|l|}{ Accès aux écrits scientifiques } \\
\hline Non & II $(17,7)$ & $29(30,5)$ & $89(29,0)$ & $138(4 \mid, 9)^{d}$ & $<0,001$ & $267(33,7)$ \\
\hline Oui (membre ACE) & $6(9,7)$ & $8(8,4)$ & $30(9,8)$ & $27(8,2)$ & 0,91 & $71(9,0)$ \\
\hline Oui, via association universitaire & $15(24,2)$ & $14(14,7)$ & $62(20,2)$ & $54(16,4)$ & 0,29 & $145(18,3)$ \\
\hline Oui, via employeur & $41(66,1)^{a,}$ e & $52(54,7)^{f}$ & $156(50,8)^{g}$ & $140(42,6)$ & 0,002 & $389(49,1)$ \\
\hline
\end{tabular}

Note. 288 participants n'ont pas indiqué à quel groupe d'âge leur principale clientèle appartenait. CRDP = Centre de réadaptation en déficience physique; CRDITED = Centre de réadaptation en déficience intellectuelle et en troubles envahissants du développement; $C P=C$ linique privée; $C L S C=C e n t r e$ local de services communautaires; $\mathrm{CH}=$ Centre hospitalier, à l'interne; $\mathrm{ACE}=$ Association canadienne des ergothérapeutes.

*Sauf lorsqu'indiqué. **Seuls les milieux de pratique et les diagnostics les plus fréquents sont présentés pour chaque clientèle. ***Les ergothérapeutes desservent des clients des deux zones.

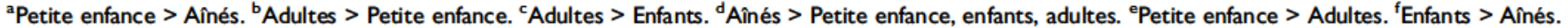

gAdultes > Aînés. 
Tableau 3

Fréquence mensuelle d’évaluation des composantes personne-environnement-occupation par clientèle

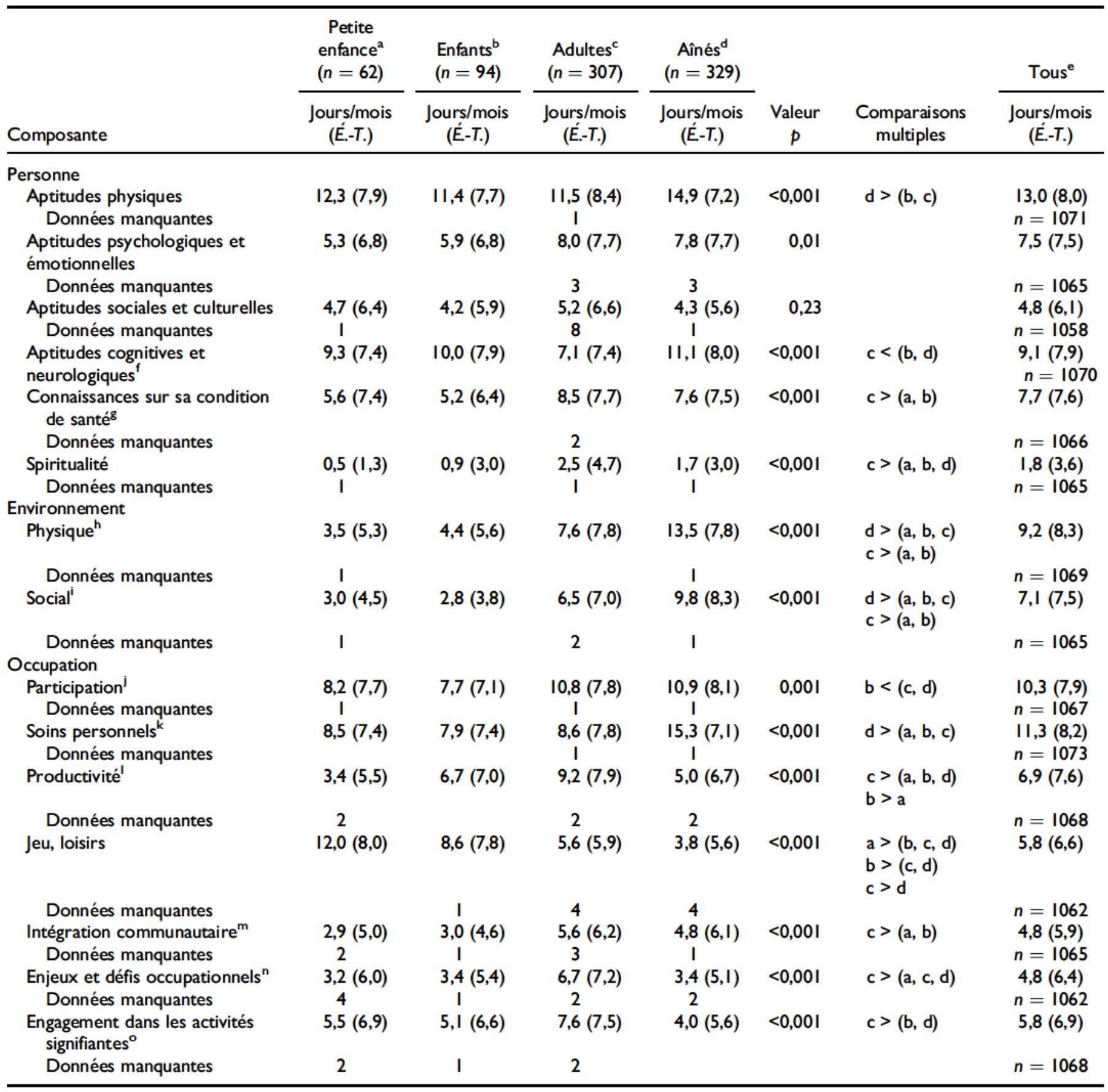

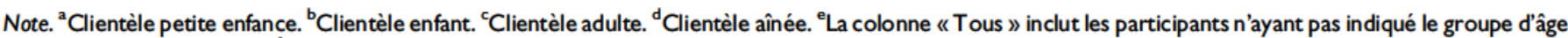
de leur principale clientèle. 'Les aptitudes cognitives et neurologiques incluent les aptitudes sensorielles et nociceptives. 'Les connaissances sur la condition de santé incluent les façons de s'améliorer et les moyens de prévenir les difficultés ou d'améliorer la fonction. 'L'environnement physique inclut l'accessibilité domiciliaire, les équipements, le poste de travail, etc. 'L'environnement social inclut le réseau, les proche-aidants, les ressources privées, le personnel du réseau public, l'employeur, l'assureur, etc. 'La participation de façon générale incluant le rendement ou la performance, la satisfaction, l'importance de ses occupations, etc. 'Les soins personnels incluent l'hygiène personnelle, l'habillage, les déplacements et transferts, etc. 'La productivité inclut le travail, le bénévolat, la gestion du foyer, les études, etc. "'L'intégration communautaire inclut la vie sociale et communautaire, les activités dans la communauté, l'utilisation des services, la conduite automobile, etc. "Les enjeux et les défis occupationnels incluent l'équilibre de vie, la routine, la privation/aliénation/transition occupationnelle, etc. 'L'engagement dans les activités signifiantes inclut les intérêts, les projets de vie et le sens des occupations. 
Tableau 4

Évaluations les plus fréquemment réalisées par mois selon les clientèles

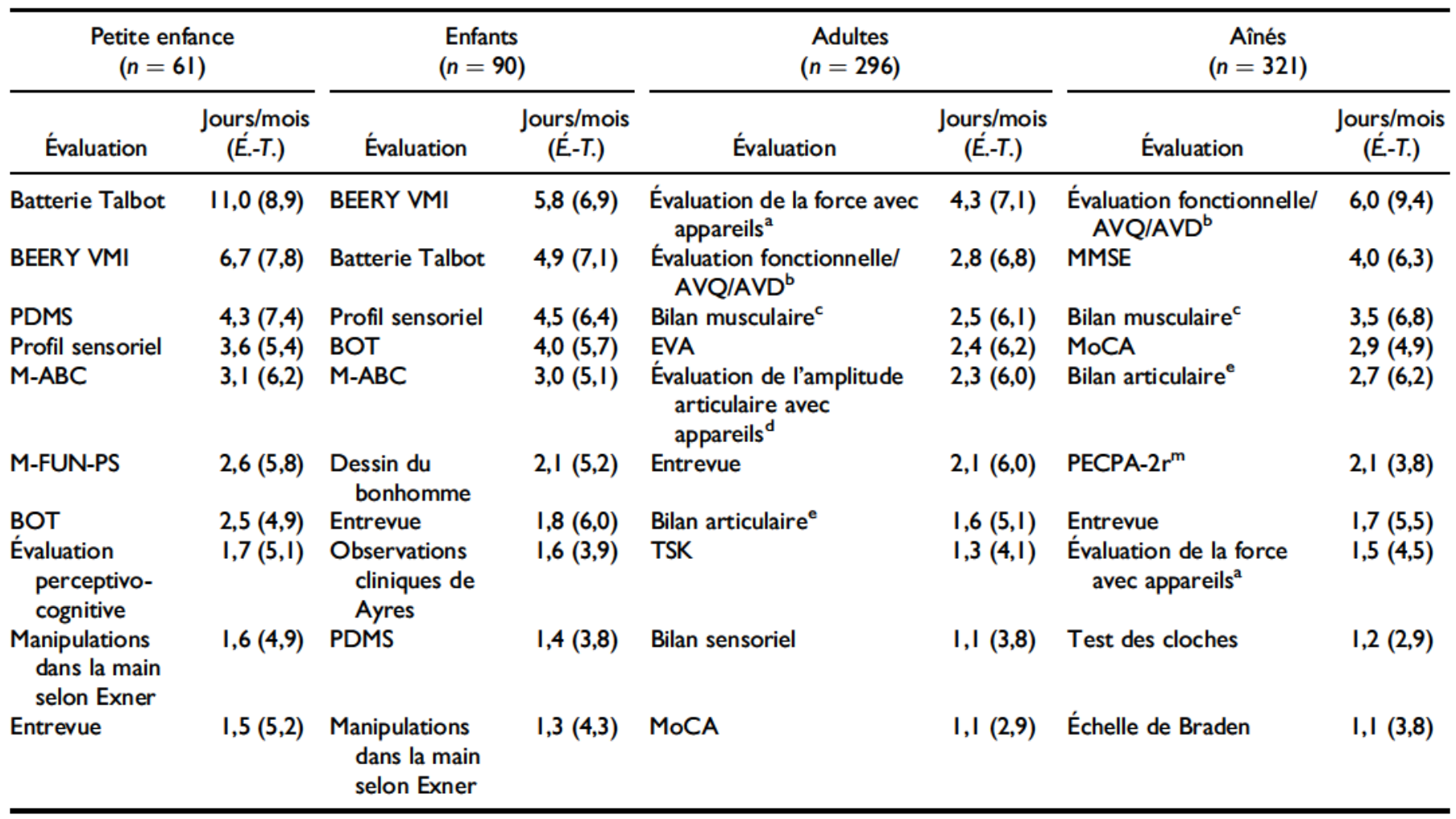

Note. BEERY VMI = Beery-Buktenica Developmental Test of Visual-Motor Integration; PDMS = Peabody Developmental Motor Scales; M-ABC = Movement Assessment Battery for Children; M-FUN-PS = Miller Function and Participation Scales; BOT = Bruininks-Oseretsky Test of Motor Proficiency; EVA = Échelle visuelle analogue pour la douleur; TSK = Échelle de kinésiophobie de Tampa; MoCA = Montreal Cognitive Assessment; MMSE = Mini-Mental State Examination; PECPA-2 $r=$ Protocole d'examen cognitif de la personne âgée.

${ }^{a}$ Inclut seulement les réponses précisant l'utilisation d'un appareil de mesure (ex. dynamomètre, pince-mètre) et n'inclut pas l'expression générale « bilan musculaire "). 'Inclut toutes les réponses en lien avec l'évaluation des activités de la vie quotidienne (AVQ), des activités de la vie domestique (AVD) et de l'aspect fonctionnel de façon générale (ex. questionnaires, mises en situation, observations et autres méthodes non spécifiées). 'Bilan musculaire fonctionnel, manuel ou «" maison "), sans précision d'utilisation d'un appareil de mesure. 'Inclut seulement les réponses précisant l'utilisation d'un appareil de mesure (ex. goniomètre,

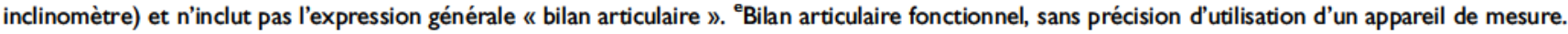

rapporté utiliser son outil associé, la Mesure canadienne du rendement occupationnel (MCRO; Law et al., 2014).

Les ergothérapeutes travaillant en petite enfance utilisaient majoritairement des outils d'évaluation reconnus et, pour la plupart, standardisés (ex. BEERY VMI, BOT, M-ABC), sauf pour le plus fréquemment utilisé, c'est-à-dire la Batterie Talbot, qui est non-standardisé (Tableau 4). Ces résultats s'appliquaient aussi à la clientèle enfant, la Batterie Talbot étant le deuxième outil d'évaluation le plus utilisé. Chez la clientèle des adultes, les outils et les méthodes d'évaluation rapportés permettaient de recueillir des données quantitatives (ex. dynamomètre, échelle visuelle analogue pour la douleur, goniomètre, etc.) et qualitatives (ex. mise en situation et observation des AVQ et AVD, entrevue; Tableau 4). Chez les aînés, les ergothérapeutes avaient davantage recours à des méthodes d'évaluation d'approche qualitative (ex. mise en situation et observation des AVQ et AVD, bilan articulaire sans outil de mesure, entrevue). Les tests de dépistage des fonctions cognitives tels que le MMSE, le MoCA et le
PECPA-2r étaient aussi plus largement utilisés auprès de cette clientèle (Tableau 4).

\section{Discussion}

Cette étude visait à dresser un portrait global des pratiques évaluatives des ergothérapeutes du Québec, et ce, pour chaque clientèle. Les pratiques évaluatives varient selon les clientèles, tant sur le plan des composantes PEO évaluées que pour les méthodes et les outils d'évaluation. À l'instar des lignes directrices de l'OEQ (OEQ, 2005a, 2005b) et considérant la diversité des clientèles et des contextes de pratique, ces résultats soutiennent l'importance de réaliser une démarche réflexive quant au choix des pratiques évaluatives des ergothérapeutes. Ces pratiques doivent non seulement être pertinentes pour la clientèle, mais aussi appropriées à leur contexte de pratique et cohérentes avec les résultats probants. 


\section{Portrait général des pratiques évaluatives des ergothérapeutes}

En concordance avec les activités professionnelles réservées aux ergothérapeutes par le Code des professions (Office des professions du Québec, 2018), les aptitudes physiques occupent généralement une grande place dans l'évaluation en ergothérapie, et ce, toute clientèle confondue. Or, l'évaluation peu fréquente des aptitudes psychologiques, émotionnelles et socioculturelles ainsi que de la spiritualité, des souscomposantes de la personne dont l'ergothérapeute doit tenir compte (OEQ, 2016b), est préoccupante. Ces résultats sont toutefois similaires à ceux d'autres études canadiennes, dont celle de Rouleau et collaborateurs (2015) issue du domaine de la santé mentale. Une autre étude réalisée auprès d'aînés en réadaptation à la suite d'un accident vasculaire cérébral (AVC) et leur proche-aidant a par ailleurs rapporté un manque de soutien psychologique (Talbot et al., 2004).

De plus, relativement peu (près du tiers) des ergothérapeutes ont rapporté utiliser des mises en situation et des observations. Pourtant, l'observation figure parmi les principales méthodes d'évaluation (OEQ, 2005b). La majorité des ergothérapeutes utiliserait ainsi plutôt des outils standardisés, des questionnaires ou des entrevues pour compléter leur évaluation. Tel que discuté par Douglas et al. (2007), en raison de la plus grande facilité à nommer un outil standardisé que de décrire une méthode d'évaluation non standardisée, les mises en situation et les observations pourraient toutefois être sous-rapportées. Les mises en situation et les observations pourraient aussi être inclues sous l'" Évaluation fonctionnelle ", une des méthodes d'évaluation les plus fréquemment rapportée chez les adultes et les aînés. Lorsque non-précisé par les ergothérapeutes, il était toutefois impossible de conclure que ces évaluations fonctionnelles impliquaient l'utilisation d'observations et de mises en situation. Or, il est possible que les outils imposés aux ergothérapeutes dans certains contextes (ex. l'Outil d'évaluation multi-clientèle) qui, d'une part, permettent la standardisation des pratiques, mais, d'autre part, limitent l'utilisation de méthodes davantage personnalisées en fonction des besoins du client (Levasseur, Carrier et Turcotte, 2016).

\section{Clientèles petite enfance et enfance}

Tel qu'observé par Saleh et collaborateurs (2008), l'outil le plus fréquemment utilisé par les ergothérapeutes en pédiatrie est la Batterie Talbot, un outil québécois dont la validation et la standardisation sont encore incomplètes (Martin, 2003). Or, l'utilisation d'outils standardisés permet notamment, et ce, pour toutes les clientèles, de restreindre les erreurs liées aux limites de la mémoire de travail lors des observations libres ainsi que de mesurer plus rigoureusement l'évolution du client (OEQ, 2014). Cette recommandation semble être malgré tout aisément suivie en pédiatrie. En effet, de façon similaire à ce qui est rapporté par d'autres études canadiennes (Brown,
Rodger, Brown et Roever, 2007; Hanna et al., 2007; Saleh et al., 2008), les outils d'évaluation rapportés en enfance étaient majoritairement standardisés. Le nombre d'heures de formation par année (presque trois fois plus élevé en petite enfance qu'en gériatrie) explique peut-être la grande utilisation d'outils standardisés en pédiatrie. Ces outils d'évaluation visent cependant surtout les aptitudes physiques et neurologiques (ex.: BEERY VMI, PDMS, M-ABC). Par ailleurs, le jeu n'est pas une cible principale de ces outils d'évaluation, mais il était la sous-composante de l'occupation la plus évaluée dans la présente étude. Ainsi, il est peut-être nécessaire que les ergothérapeutes intègrent de nouvelles méthodes ou outils d'évaluation de façon à ce que le jeu, une occupation importante en pédiatrie, soit mieux inclus dans l'évaluation ergothérapique.

\section{Clientèle adulte}

Les pratiques évaluatives rapportées par les ergothérapeutes travaillant auprès de la clientèle adulte visaient surtout les aptitudes physiques (mesures de la force et de l'amplitude articulaire, bilan sensoriel), la douleur (échelle visuelle analogue pour la douleur) ainsi que le fonctionnement dans les AVQ. Une collecte de données précises et mesurables sur les aptitudes de la personne concorde avec les lignes directrices des guides de pratique de l'OEQ pour l'évaluation du fonctionnement au travail (OEQ, 2016a), c'est-à-dire la productivité, la deuxième sous-dimension de l'occupation la plus évaluée. Afin de trianguler les données recueillies, l'utilisation de méthodes qualitatives telles que l'observation du fonctionnement dans des activités ainsi que l'entrevue (rapportées dans le sondage) sont également recommandées. Étant une occupation importante et déterminante pour une grande partie de cette clientèle, il est logique que les pratiques évaluatives des ergothérapeutes se concentrent autour du travail. Or, dans la présente étude, la participation était la sous-composante de l'occupation la plus évaluée, mais les méthodes d'évaluation y étaient peu liées. Ainsi, un outil directement en lien avec la participation pourrait être, par exemple, la MCRO (Law et al., 2014).

\section{Clientèle aînée}

De façon similaire à l'étude de Veillette et collaborateurs (2007) décrivant la pratique des ergothérapeutes à l'urgence, l'évaluation ergothérapique au Québec auprès des aînés porte essentiellement sur les aptitudes physiques, le dépistage des difficultés cognitives et le fonctionnement sécuritaire dans les AVQ. Ces pratiques évaluatives, qui rejoignent les trois composantes théoriques (PEO), sont importantes pour le maintien à domicile. Cependant, il est aussi important de ne pas restreindre l'évaluation aux activités quotidiennes de base, telles que les soins personnels, et de porter attention aux activités sociales. En effet, selon une étude réalisée auprès d'aînés ayant des incapacités et recevant des services du soutien à domicile, plusieurs besoins non satisfaits 
concernent les activités sociales et de loisirs (Levasseur et al., 2014). Ces besoins sont d'ailleurs moins évalués, notamment en raison des contraintes du contexte de pratique des intervenants. Ces contraintes incluent l'utilisation d'outils de référencement (Carrier, Freeman, Levasseur et Desrosiers, 2015) et d'évaluation systématiques (Levasseur et al., 2016), qui laissent peu de place à évaluer de façon exhaustive et audelà de l'autonomie aux activités de la vie quotidienne. Les écrits scientifiques mettent en lumière l'importance d'utiliser des outils d'évaluation centrés sur le client (Talbot et al., 2004; Townsend et Polatakjo, 2013;) et sur l'occupation (Levasseur et al., 2016), tels que la MCRO, qui pourraient être utilisés de façon répétée au cours de la trajectoire de soins de l'aîné, et ce, afin de mettre les besoins qu'il a identifié au premier plan. Bien qu'elle semble être un outil fréquemment utilisé en santé mentale au Canada (Rouleau et al., 2015), la MCRO a été peu rapportée dans la présente étude $(n=61 ; 7,0 \%)$. Or, en plus d'être accessible, facile et rapide à administrer (Law et al., 2014), la MCRO a été validée auprès d'aînés ayant divers diagnostics (Chan et Lee, 1997; McNulty et Beplat, 2008; Thyer, Brown et Roe, 2018) et fait partie des outils recommandés par les guides de pratique pour l'évaluation ergothérapique de la clientèle gériatrique (Bortnick, 2017). Cependant, le besoin d'être formé et de se procurer le matériel requis pour utiliser la MCRO (http:// www.thecopm.ca/) peuvent être des obstacles, considérant le nombre limité d'heures de formation et l'accès plus difficile aux écrits scientifiques en gériatrie, comparativement à ceux rapportés par les ergothérapeutes travaillant en petite enfance.

\section{Forces et limites de l'étude}

Cette étude est la première, à notre connaissance, ayant permis de documenter les pratiques évaluatives des ergothérapeutes du Québec, et ce, selon toutes les clientèles. Par contre, cette étude ne présente pas d'analyse par type de mission d'établissement (ex. CLSC, CHSLD, etc.), Or, les mandats des ergothérapeutes et les composantes pertinentes à évaluer chez leur clientèle varient selon la mission dans laquelle ils œuvrent. De même, la répartition des tâches d'évaluation entre les professionnels d'une équipe varie selon l'organisation propre au contexte de pratique. Ainsi, même si elles ne sont pas directement évaluées par l'ergothérapeute, les composantes évaluées par d'autres membres de l'équipe peuvent être considérées dans l'analyse occupationnelle, ce qui n'a pas été considéré dans la présente étude. De plus, puisqu'il s'appuie sur un questionnaire autorapporté, ce portrait représente la perception des ergothérapeutes quant à leur pratique. Ainsi, il peut être difficile pour les répondants de rapporter précisément leurs fréquences ainsi que leurs méthodes et leurs outils d'évaluation. Malgré une classification rigoureuse et validée par des experts, la grande variété et le manque de précision de certaines réponses ont rendu difficile la reconnaissance et la catégorisation adéquate des méthodes et des outils d'évaluation rapportés, ce qui peut avoir eu pour effet de réduire certaines prévalences. Enfin, malgré la consigne de rapporter le plus fidèlement possible les méthodes et les outils d'évaluation qu'ils utilisent dans leur pratique, un biais de désirabilité sociale pourrait avoir influencé les réponses des ergothérapeutes.

\section{Conclusion}

L'objectif de cette étude était de dresser un portrait global des pratiques évaluatives des ergothérapeutes du Québec, et ce, pour chaque clientèle, c'est-à-dire la petite enfance, les enfants, les adultes et les aînés. Les ergothérapeutes ont rapporté des pratiques généralement diversifiées selon les clientèles, particulièrement en ce qui concerne les souscomposantes de l'occupation évaluées ainsi que les méthodes et les outils d'évaluation. Globalement, l'évaluation est très axée sur les aptitudes physiques, et ce, pour toutes les clientèles, alors que l'environnement et l'occupation mériteraient une plus grande attention. Sachant que l'ergothérapeute a une vision holistique de l'humain comme être occupationnel (Townsend et Polatakjo, 2013), l'évaluation des aptitudes physiques pourrait potentiellement être davantage réalisée par d'autres collègues, notamment les physiothérapeutes, afin que les ergothérapeutes puissent apprécier les autres sous-composantes de la personne, telles que les aptitudes psychologiques, émotionnelles et spirituelles, et évaluer les enjeux liés à l'occupation et à l'environnement. D'ailleurs, afin de mettre l'occupation au premier plan, de plus en plus d'outils documentant les défis occupationnels, tels que l'équilibre de vie (Larivière et Levasseur, 2016) et les occupations porteuses de sens (Lacroix et al., 2018), sont disponibles et seraient bénéfiques à utiliser. Toutefois, dans le cadre de certains contextes et mandats, l'évaluation exhaustive du client sous toutes ses composantes n'est pas possible ou pertinente. Ainsi, une analyse plus approfondie identifiant plus spécifiquement les écarts entre les pratiques évaluatives et les résultats probants selon les différentes clientèles et contextes de pratique doit se poursuivre. Afin, ultimement, d'identifier les changements souhaitables pour une pratique davantage fondée sur les résultats probants, il importe également d'approfondir le pourquoi et le comment de ces pratiques.

\section{Messages clés}

- Les aptitudes physiques sont la sous-composante de la personne la plus évaluée, alors que la spiritualité ainsi que les aptitudes psychologiques, émotionnelles, sociales et culturelles sont sous-évaluées, et ce, pour toutes les clientèles.

- Une évaluation holistique incluant toutes les composantes du PEO est recommandée, surtout chez les enfants et les adultes.

- Il est nécessaire d'utiliser des outils évaluant l'occupation, notamment pour s'assurer d'identifier et d'intervenir sur les activités les plus signifiantes pour le client. 


\section{ORCID iD}

Janie Gobeil (D) https://orcid.org/0000-0002-5353-4247

\section{Apport des auteurs}

Janie Gobeil : Recension des écrits, analyse et interprétation des données et rédaction du manuscrit.

Nadine Larivière : Participation à l'élaboration du sondage, analyse des données et révision du manuscrit.

Annie Carrier : Participation à l'élaboration du sondage, analyse des données et révision du manuscrit.

Nathalie Bier : Participation à l'élaboration du sondage, analyse des données et révision du manuscrit.

Carolina Bottari : Participation à l'élaboration du sondage, analyse des données et révision du manuscrit.

Nathalie Veillette : Participation à l'élaboration du sondage, analyse des données et révision du manuscrit.

Suzanne Rouleau : Participation à l'élaboration du sondage, analyse des données et révision du manuscrit.

Isabelle Gélinas : Participation à l'élaboration du sondage, analyse des données et révision du manuscrit.

Véronique Provencher : Participation à l'élaboration du sondage, analyse des données et révision du manuscrit.

Mélanie Couture : Participation à l'élaboration du sondage, analyse des données et révision du manuscrit.

Mélanie Levasseur : Direction du projet, rédaction et réalisation du sondage, analyse des données, rédaction et révision du manuscrit.

\section{Références}

Association canadienne des ergothérapeutes. (2012). Profil de la pratique des ergothérapeutes au Canada. Repéré à https:// www.caot.ca/document/4720/2012profil.pdf

Belchior, P., Korner-Bitensky, N., Holmes, M., et Robert, A. (2015). Identification and assessment of functional performance in mild cognitive impairment: A survey of occupational therapy practices. Australian Occupational Therapy Journal, 62(3), 187-196. doi:10. 1111/1440-1630.12201

Bortnick, K. (2017). Occupational therapy assessment for older adults: 100 instruments for measuring occupational performance. Thorofare, NJ: Slack. Repéré à http://ezproxy.usherbrooke.ca/ login?url=https://search.ebscohost.com/login.aspx?direct= true $\& \mathrm{db}=$ nlebk \&AN $=1462586 \& \&$ site $=$ eds-live

Brown, G. T., Rodger, S., Brown, A., et Roever, C. (2007). A profile of Canadian pediatric occupational therapy practice. Occupational Therapy in Health Care, 21(4), 39-69. doi:10.1080/J003v21n04_03

Carrier, A., Freeman, A., Levasseur, M., et Desrosiers, J. (2015). Standardised referral form: Restricting client-centred practice? Scandinavian Journal of Occupational Therapy, 22(4), 283-292. doi:10.3109/1 1038128.2015.1019922

Chan, C. C. H., et Lee, T. M. C. (1997). Validity of the Canadian Occupational Performance Measure. Occupational Therapy International, 4(3), 231-249.

Douglas, A., Liu, L., Warren, S., et Hopper, T. (2007). Cognitive assessments for older adults: Which ones are used by Canadian therapists and why. Canadian Journal of Occupational Therapy, 74(5), 370-381. doi: 10.2182/cjot.07.010

Fougeyrollas, P. (2010). La funambule, le fil et la toile: transformations réciproques du sens du handicap. Québec, QC: Presses de l'Université Laval.

Hanna, S. E., Russell, D. J., Bartlett, D. J., Kertoy, M., Rosenbaum, P. L., et Wynn, K. (2007). Measurement practices in pediatric rehabilitation: A survey of physical therapists, occupational therapists, and speech-language pathologists in Ontario. Physical \& Occupational Therapy in Pediatrics, 27(2), 25-42. doi:10.1080/ J006v27n02_03

Institut canadien d'information sur la santé. (26 novembre 2015). Les ergothérapeutes 2014. Repéré à https://secure.cihi.ca/estore/pro ductFamily.htm?locale $=$ fr \&pf $=$ PFC3040\&

Korner-Bitensky, N., Barrett-Bernstein, S., Bibas, G., et Poulin, V. (2011). National survey of Canadian occupational therapists' assessment and treatment of cognitive impairment post-stroke. Australian Occupational Therapy Journal, 58(4), 241-250. doi: 10.1111/j.1440-1630.2011.00943.x

Lacroix, P.-A., Pelletier, A.-J., Blondin, M.-P., Dugal, A., Langlois, C., Levasseur, M., et Larivière, N. (2018). Traduction et validation du Questionnaire sur l'engagement dans les activités signifiantes. Canadian Journal of Occupational Therapy, 85(1), 11-20. doi:10. 1177/0008417417702925

Larivière, N., et Levasseur, M. (2016). Traduction et validation du questionnaire ergothérapique l'Inventaire de l'équilibre de vie. Canadian Journal of Occupational Therapy, 83(2), 103-114. doi: $10.1177 / 0008417416632260$

Law, M., Baptiste, S., Carswell, A., McColl, M. A., Polatajko, H., et Pollock, N. (2014). MCRO. La mesure canadienne du rendement occupationnel (5e éd.). Ottawa, ON: CAOT Publications ACE.

Levasseur, M., Carrier, A., et Turcotte, P.-L. (2016). Réflexion sur l'utilisation de l'Outil d'évaluation Multiclientèle (OÉMC) pour identifier les besoins de participation sociale des aînés ayant des incapacités. Développement humain, handicap et changement social, 22(1), 23-55.

Levasseur, M., Larivière, N., Royer, N., Desrosiers, J., Landreville, P., Voyer, P., ... Sévigny, A. (2014). Match between needs and services for participation of older adults receiving home care: Appraisals and challenges. Leadership in Health Services, 27(3), 204-223. doi:10.1108/LHS-072013-0030

Martin, M.-E. (2003). Batterie d'évaluation du développement Talbot pour l'enfant de 0 à 6 ans: validité de contenu par analyse des items (Mémoire de maîtrise). Repéré à https://papyrus.bib.umon treal.ca/xmlui/handle/1866/14483? show=full

McNulty, M. C., et Beplat, A. L. (2008). The validity of using the Canadian Occupational Performance Measure with older adults with and without depressive symptoms. Physical \& Occupational Therapy In Geriatrics, 27(1), 1-15. doi:10.1080/02703180 802206231

Office des professions du Québec. (2018, juin). Code des professions. Repéré à http://legisquebec.gouv.qc.ca/fr/pdf/cs/C26.pdf 
Ordre des ergothérapeutes du Québec. (2005a). Choisir les instruments de mesure: impact sur l'analyse des résultats de l'évaluation. Ergothérapie express-Le bulletin de l'Ordre des ergothérapeutes du Québec, 16(3), 6. Repéré à https://www.oeq.org/publications/ ergotherapie-express/articles-sur-la-pratique-professionnelle.html

Ordre des ergothérapeutes du Québec. (2005b). Choisir les instruments de mesure: une responsabilité de l'ergothérapeute. Ergothérapie express-Le bulletin de l'Ordre des ergothérapeutes du Québec, 16(2), 4. Repéré à https://www.oeq.org/pu blications/ergotherapie-express/articles-sur-la-pratiqueprofessionnelle.html

Ordre des ergothérapeutes du Québec. (2010). Référentiel de compétences lié à l'exercice de la profession d'ergothérapeute au Québec (Mise à jour 2013). Repéré à https://www.oeq.org/ DATA/NORME/13 v referentiel-de-competences_2013_ couleurs.pdf

Ordre des ergothérapeutes du Québec. (2014). Justesse et pertinence de l'évaluation: l'importance de l'utilisation d'instruments de mesure éprouvés. Ergothérapie express-Le bulletin de l'Ordre des ergothérapeutes du Québec, 25(4), 5. Repéré à https://www.oe q.org/publications/ergotherapie-express/articles-sur-la-pratiqueprofessionnelle.html

Ordre des ergothérapeutes du Québec. (2016a). Évaluation du fonctionnement au travail des personnes ayant une déficience physique: Guide de l'ergothérapeute. Repéré à https://www.oeq.org/ DATA/NORME/11 v guideevalfonctdefphys.pdf

Ordre des ergothérapeutes du Québec. (2016b). L'utilisation de modèles et d'approches propres à notre profession: faire ressortir notre spécificité d'ergothérapeute. Ergothérapie express-Le bulletin de l'Ordre des ergothérapeutes du Québec, 27(4), 6. Repéré à https:// www.oeq.org/publications/ergotherapie-express/articles-sur-la-pra tique-professionnelle.html

Richard, L., Gauvin, L., Gosselin, C., et Laforest, S. (2008). Staying connected: Neighbourhood correlates of social participation among older adults living in an urban environment in Montréal, Québec. Health Promotion International, 24(1), 46-57. doi:10. 1093/heapro/dan039

Rouleau, S., Dion, K., et Korner-Bitensky, N. (2015). Assessment practices of Canadian occupational therapists working with adults with mental disorders. Canadian Journal of Occupational Therapy, 82(3), 181-193. doi:10.1177/0008417414561857

Saleh, M. N., Korner-Bitensky, N., Snider, L., Malouin, F., Mazer, B., Kennedy, E., et Roy, M.-A. (2008). Actual vs. best practices for young children with cerebral palsy: A survey of paediatric occupational therapists and physical therapists in Quebec, Canada. Developmental Neurorehabilitation, 11(1), 60-80. doi:10.1080/ 17518420701544230.

Talbot, L., Viscogliosi, C., Desrosiers, J., Vincent, C., Rousseau, J., et Robichaud, L. (2004). Identification of rehabilitation needs after a stroke: an exploratory study. Health and Quality of Life Outcomes, 2(53), 1-9. doi:10.1186/1477-7525-2-53

Townsend, E. A., et Polatajko, H. (2013). Enabling occupation II: Advancing an occupational therapy vision for health, wellbeing, \& justice through occupation. Ottawa, ON: CAOT Publishers.
Thyer, L., Brown, T., et Roe, D. (2018). The validity of the Canadian Occupational Performance Measure (COPM) when used in a subacute rehabilitation setting with older adults. Occupational Therapy in Health Care, 32(2), 137-153. doi:10.1080/07380577.2018. 1446233

Veillette, N., Demers, L., et Dutil, É. (2007). Description de la pratique des ergothérapeutes du Québec en salle d'urgence. Revue canadienne d'ergothérapie, 74(4), 348-358. doi:10.2182/cjot.07. 006

\section{Biographies des auteures}

Janie Gobeil, M. Erg., est étudiante à la maîtrise, Faculté de médecine et des sciences de la santé, Université de Sherbrooke, QC, Canada, et auxiliaire de recherche, Centre de recherche sur le vieillissement du CIUSSS de l'Estrie-CHUS, Sherbrooke, QC, Canada. Au moment de la rédaction de ce manuscrit, Janie Gobeil était étudiante à la maîtrise au programme d'ergothérapie de l'Université de Sherbrooke.

Nadine Larivière, $\mathrm{PhD}$, erg., est professeure agrégée, et directrice du programme d'ergothérapie, École de réadaptation, Université de Sherbrooke, QC, Canada, et chercheure, Institut universitaire de première ligne en santé et services sociaux, Sherbrooke, QC, Canada.

Annie Carrier, $\mathrm{PhD}$, erg., est professeure adjointe, École de réadaptation, Université de Sherbrooke, QC, Canada, et chercheure, Centre de recherche sur le vieillissement du CIUSSS de l'Estrie-CHUS, Sherbrooke, QC, Canada.

Nathalie Bier, $\mathrm{PhD}$, erg., est professeure agrégée, École de réadaptation, Université de Montréal, QC, Canada, et chercheure, Centre de recherche de l'Institut universitaire de gériatrie de Montréal, QC, Canada.

Carolina Bottari, $\mathrm{PhD}$, erg., est professeure agrégée, École de réadaptation, Université de Montréal, QC, Canada, et chercheure, Centre de recherche interdisciplinaire en réadaptation du Montréal métropolitain, Montréal, QC, Canada.

Nathalie Veillette, $\mathrm{PhD}$, erg., est professeure agrégée, École de réadaptation, Université de Montréal, QC, Canada, et chercheure, Centre de recherche de l'Institut universitaire de gériatrie de Montréal, QC, Canada.

Suzanne Rouleau, MSc, erg., est professeure adjointe, École de physiothérapie et d'ergothérapie, Université McGill, Montréal, QC, Canada.

Isabelle Gélinas, $\mathrm{PhD}$, erg., est professeure agrégée, École de physiothérapie et d'ergothérapie, Université McGill, Montréal, QC, Canada, et chercheure, Centre de recherche interdisciplinaire en réadaptation du Montréal métropolitain, Montréal, QC, Canada. 
Véronique Provencher, $\mathrm{PhD}$, erg., est professeure adjointe, École de réadaptation, Université de Sherbrooke, QC, Canada, et chercheure, Centre de recherche sur le vieillissement du CIUSSS de l'Estrie-CHUS, Sherbrooke, QC, Canada.

Mélanie Couture, $\mathrm{PhD}$, erg., est professeure agrégée, École de réadaptation, Université de Sherbrooke, QC, Canada, et chercheure, Centre de recherche du Centre hospitalier universitaire de Sherbrooke, QC, Canada.

Mélanie Levasseur, $\mathrm{PhD}$, erg., est professeure agrégée, École de réadaptation, Université de Sherbrooke, QC, Canada, et chercheure, Centre de recherche sur le vieillissement du CIUSSS de l'Estrie-CHUS, Sherbrooke, QC, Canada. 